\title{
New and Improved Criteria on Fundamental Properties of Solutions of Integro-Delay Differential Equations with Constant Delay
}

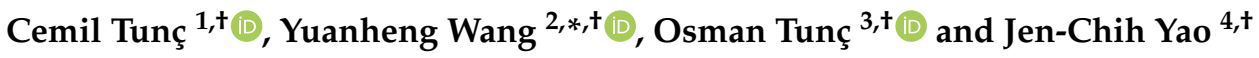 \\ 1 Department of Mathematics, Faculty of Sciences, Van Yuzuncu Yil University, Van 65080, Turkey; \\ cemtunc@yahoo.com \\ 2 Department of Mathematics, Zhejiang Normal University, Jinhua 321004, China \\ 3 Department of Computer Programing, Baskale Vocational School, Van Yuzuncu Yil University, \\ Van 65080, Turkey; osmantunc89@gmail.com \\ 4 Research Center for Interneural Computing, China Medical University Hospital, China Medical University, \\ Taichung 404332, Taiwan; yaojc@mail.cmu.edu.tw \\ * Correspondence: yhwang@zjnu.cn \\ + These authors contributed equally to this work.
}

Citation: Tunç, C.; Wang, Y.; Tunç, O.; Yao, J.-C. New and Improved

Criteria on Fundamental Properties of Solutions of Integro-Delay Differential Equations with Constant Delay. Mathematics 2021, 9, 3317.

https://doi.org/10.3390/

math 9243317

Academic Editor:Alberto Ferrero

Received: 16 November 2021

Accepted: 14 December 2021

Published: 20 December 2021

Publisher's Note: MDPI stays neutral with regard to jurisdictional claims in published maps and institutional affiliations.

Copyright: (c) 2021 by the authors. Licensee MDPI, Basel, Switzerland. This article is an open access article distributed under the terms and conditions of the Creative Commons Attribution (CC BY) license (https:// creativecommons.org/licenses/by/ $4.0 /)$.

\begin{abstract}
This paper is concerned with certain non-linear unperturbed and perturbed systems of integro-delay differential equations (IDDEs). We investigate fundamental properties of solutions such as uniformly stability (US), uniformly asymptotically stability (UAS), integrability and instability of the un-perturbed system of the IDDEs as well as the boundedness of the perturbed system of IDDEs. In this paper, five new and improved fundamental qualitative results, which have less conservative conditions, are obtained on the mentioned fundamental properties of solutions. The technique used in the proofs depends on Lyapunov-Krasovskir functionals (LKFs). In particular cases, three examples and their numerical simulations are provided as numerical applications of this paper. This paper provides new, extensive and improved contributions to the theory of IDDEs.
\end{abstract}

Keywords: non-linear system; IDDEs; constant delay; LKF; stability; instability; integrability; boundedness

MSC: 93D20; 26D15; 34K06; 24K20

\section{Introduction}

Integral equations (IEs) and integro-differential equations (IDEs) occur widely in diverse areas of applied mathematics and physics. IEs and IDEs offer powerful techniques to solve various practical problems in numerous different scientific fields, and these equations have also attracted attentions for these reasons by numerous researchers up to now. Indeed, IDEs appear when we convert initial value problems to IEs. During the last a few decades, there has been much work on fundamental properties of solutions of IDEs and IDDEs by using the LKF method, the Jensen inequality method, the fixed point method, the variation of parameters formula, Wirtinger-based integral inequality, the free-weighting matrix method and so on (see, for example, [1-21]). Among these methods, the LKF method can be used very effectively during the investigation of the fundamental properties solutions of IDEs and IDDEs. The positive effects of the LKF method depend on construction or choice or definition of a suitable LKF. Indeed, the major advantage of the LKF method is that the stability in the large can be obtained without any prior knowledge of solutions. Without loss of generality, during the investigations, the LKF method requires the construction or definition of suitable functional(s) as a basic tool , known as the Lyapunov-Krasovskir functional(s), to prove qualitative results on fundamental properties of solutions, stability, 
instability, boundedness, convergence, etc., of FDEs, IDDE, and so on. The major disadvantage of this method is that constructing or defining suitable LKFs remains an open problem in the literature up to now. However, when a suitable LKF is found, the LKF method is very effective to obtain qualitative results under weaker and effective conditions. In this paper, we will use the LKF method as a basic tool for problems to be considered here.

First, we would like to present some recent works related to this paper.

In 2020, Zeng et al., [20] studied the asymptotic stability (AS) of the delay linear system:

$$
\dot{x}(t)=A x(t)+A_{1} x(t-h)+A_{2} \int_{t-h}^{t} x(s) d s,
$$

where $x(t) \in \mathbb{R}^{n}, t \in[-h, \infty), x(t)=\phi(t)$ on $[-h, 0], A, A_{1}, A_{2} \in \mathbb{R}^{n \times n}$ and $h$ is the constant time retardation. In [20], the new integral inequality was devised and used with the LKF to investigate the AS of the above delay linear system.

In 2020, Tian et al., [10] considered the delay linear system given by

$$
\dot{x}(t)=A x(t)+B x(t)+C \int_{t-h}^{t} x(s) d s,
$$

where $x(t) \in \mathbb{R}^{n}$ is the sate vector, $t \in[-h, \infty), x(t)=\phi(t)$ on $[-h, 0]$ and $A, B, C \in \mathbb{R}^{n \times n}$. Tian et al., [10] proved the AS of the above system. In [10], the new integral inequality is obtained. Later, the delay-dependent stability criteria are established using this inequality and the LKF.

In 2020, Tian and Wang [11] investigated the AS of the following delay linear system:

$$
\dot{x}(t)=A x(t)+A_{d} x(t-h)+A_{D} \int_{t-h}^{t} x(s) d s,
$$

where $x(t) \in \mathbb{R}^{n}$ is the sate vector, $t \in[-h, \infty), x(t)=\phi(t)$ on $[-h, 0], A, A_{d}, A_{D} \in \mathbb{R}^{n \times n}$ and $h$ is the constant delay. In [11], firstly, the vectors-based multiple integral inequality is constructed. Then, based on this integral inequality and the LKF, new delay-dependent stability criteria are derived for the above system.

In two nice papers published recently, Liu and Zhao [7] and Zhao et al., [18] investigated (AS) of the linear system of IDDEs with state and distributed delays:

$$
\dot{x}(t)=A x(t)+A_{d} x(t-h)+A_{D} \int_{t-h}^{t} x(s) d s,
$$

where $x(t) \in \mathbb{R}^{n}, A, A_{d}, A_{D} \in \mathbb{R}^{n \times n}$ are constant matrices, $x(t)=\phi(t)$ on $[-h, 0], \phi \in$ $C\left([-h, 0], \mathbb{R}^{n}\right)$ is an initial function and $h$ is the constant delay.

In Zhao et al., [18], Theorem 1, first the double integral inequality was defined by the authors. Then, the proposed double integral inequality was used to derive new delaydependent stability criteria by using LMIs (linear matrix inequalities) to guarantee the AS of system (1). In [18], two numerical examples were also given to demonstrate the effectiveness and merits of the AS result.

When we look at the above delay systems all of them are linear and have simple forms. Indeed, the result of the paper [18] is very nice and interesting. To the best of knowledge, the AS result of this paper has conservative conditions because of the LKF used therein. 
In this paper, particularly motivated from the result of Zhao et al., [18], Theorem 1 and the works mentioned above, we consider the following perturbed nonlinear system of IDDEs with state and distributed delays:

$$
\dot{x}(t)=A(t) x(t)+A_{d}(t) F(x(t-h))+\int_{t-h}^{t} A_{D}(s) G(x(s)) d s+H(t, x(t), x(t-h)),
$$

where $x(t) \in \mathbb{R}^{n}, t \in[-h, \infty), x(t)=\phi(t)$ on $[-h, 0], h \in \mathbb{R}, h>0$ is delay term, $\phi \in C\left([-h, 0], \mathbb{R}^{n}\right)$ is the initial function, $A(t)=\left(a_{i j}(t)\right), A_{d}=\left(a_{d i j}(t)\right), A(t), A_{d}(t) \in$ $C\left[\mathbb{R}^{+}, \mathbb{R}^{n \times n}\right], \mathbb{R}^{+}=[0, \infty), A_{D}=\left(a_{D i j}(s)\right), i, j=1,2, \ldots, n,-h \leq s \leq t<\infty, A_{D} \in$ $C\left[[-h, \infty), \mathbb{R}^{n \times n}\right], F, G \in C\left[\mathbb{R}^{n}, \mathbb{R}^{n}\right], F(0)=G(0)=0$ and $H \in C\left(\mathbb{R}^{+} \times \mathbb{R}^{n} \times \mathbb{R}^{n}, \mathbb{R}^{n}\right)$.

Since the functions $F, G$ and $H$ are continuous, the continuity of these functions is a sufficient conditions for the existence of the solutions of the system of IDDEs (2). We also assume that the functions $F, G$ and $H$ satisfy the Lipschitz condition in their respective arguments $x(t-h), x$ and $x, x(t-h)$, respectively. This assumption is a sufficient condition for the uniqueness of the solutions of the system of IDDEs (2).

It is obvious that the system of IDDEs (2) includes all of the above systems and extends these systems from the linear case to a very general nonlinear delay system. Next, in the mentioned papers, it was focused on the maximum admissible upper bounds of the delay term. At the end of this paper, we also give some comments on the upper bounds of the delay term.

Here, in particular, we focus on the paper of Zhao et al., [18] with some details. The aim of the paper is to obtain the result of [18] under weaker conditions, to extend and improve it. We also added four new results with some numerical simulations. The information given above shortly outline the novelty, originality and contributions of this paper.

We should also mention that if we replace $h$ by $-h$ in the perturbed nonlinear system of IDDEs (2), then we will obtain an IDE with advanced argument. To the best of information, there is no work in the literature on the fundamental properties of solutions of IDEs with advanced arguments. This is an open problem and this work could be extended for the advanced case in the future.

The remainder of this paper is organized as follows: Section 2 includes the preliminaries such as background information of the paper and the result of Zhao et al., [18], Theorem 1. Section 3 includes the main results of this paper, the stability and integrability results, Theorems $2-4$. In Section 4, an illustrative example is given to support our new results, Theorems 2-4. In Sections 5 and 6, the instability theorem, Theorem 5 and the related illustrative example, Example 2, are provided, respectively. Similarly, in Sections 7 and 8, the boundedness theorem, Theorem 6, and an illustrative example, Example 3, are given, respectively. Finally, Sections 9 and 10 include the contributions and the conclusion of the paper, respectively

\section{Preliminaries}

We begin by couching our problem in a more general setting. Consider the system of functional differential equations (FDEs) (see, Burton [3]):

$$
\frac{d x}{d t}=G\left(t, x_{t}\right)
$$

with $G: \mathbb{R}^{+} \times C \rightarrow \mathbb{R}^{n}$ being continuous, where $\mathbb{R}^{+}=[0, \infty), \mathbb{R}=(-\infty, \infty)$, and $C$ is the set of continuous functions $\phi:[-\tau, 0] \rightarrow \mathbb{R}^{n}, \tau>0$. We suppose that $G$ takes closed bounded sets of $\mathbb{R}^{+} \times C$ into bounded sets in $\mathbb{R}^{n}$.

As the next step, for any $a \geq 0, t_{0} \geq 0$, and $x \in C\left(\left[t_{0}-\tau, t_{0}+a\right], \mathbb{R}^{n}\right)$, we assume that $x_{t}=x(t+\theta)$ for $\theta \in[-\tau, 0]$. 
For $x \in \mathbb{R}^{n}$, the norm $\|\cdot\|$ is denoted by

$$
\|x\|=\sum_{i=1}^{n}\left|x_{i}\right| .
$$

If $A \in \mathbb{R}^{n \times n}$, then, $\|A\|$ is defined by

$$
\|A\|=\max _{1 \leq j \leq n}\left(\sum_{i=1}^{n}\left|a_{i j}\right|\right)
$$

For any $\phi \in C$, let

$$
\|\phi\|_{C}=\sup _{\theta \in[-\tau, 0]}\|\phi(\theta)\|=\|\phi(\theta)\|_{[-\tau, 0]}
$$

and

$$
C_{H}=\left\{\phi: \phi \in C \text { and }\|\phi\|_{C} \leq H<\infty\right\} .
$$

Throughout this paper, we tacitly assume that the function $G$ satisfies conditions which guarantee the uniqueness of solutions of system of FDEs (3). We should point out that IDDEs (1) and (2) are included by system of FDEs (3).

We suppose that $x(t)=x\left(t, t_{0}, \phi\right)$ is a solution of system of FDEs (3) with $x(t)=\phi(t)$ on $\left[t_{0}-\tau, t_{0}\right]$.

Zhao et al., [18], Theorem 1 proved the following theorem.

Theorem 1. (Zhao et al., [18], Theorem 1). Given $h>0$, system (1) is AS if there exist positive definite matrices $P \in \mathbb{R}^{4 n \times 4 n}$ and $Q, S, R \in \mathbb{R}^{n \times n}$ such that the following LMI holds:

$$
\begin{aligned}
\Psi= & \operatorname{sym}\left(\Pi_{1}^{T} P \Pi_{2}\right)+e_{1}^{T} Q e_{1}-e_{2}^{T} Q e_{2}+h^{2} e_{0}^{T} S e_{0}+\frac{h^{2}}{2} e_{0}^{T} \operatorname{Re}_{0}-\Pi_{3}^{T} S \Pi_{3} \\
& -3 \Pi_{4}^{T} S \Pi_{4}-5 \Pi_{5}^{T} S \Pi_{5}-2 \Pi_{6}^{T} R \Pi_{6}-4 \Pi_{7}^{T} R \Pi_{7}-6 \Pi_{8}^{T} R \Pi_{8}<0,
\end{aligned}
$$

where

$$
\begin{gathered}
\Pi_{1}=\left[e_{1}^{T}, e_{3}^{T}, e_{4}^{T}, e_{5}^{T}\right]^{T}, \\
\Pi_{2}=\left[e_{3}^{T}, e_{1}^{T}-e_{2}^{T}, h e_{1}^{T}-e_{3}^{T}, \frac{h^{2}}{2} e_{1}^{T}-e_{4}^{T}\right]^{T}, \\
\Pi_{3}=e_{1}-e_{2}, \\
\Pi_{4}=e_{1}+e_{2}-\frac{2}{h} e_{3}, \\
\Pi_{5}=e_{1}-e_{2}+\frac{6}{h} e_{3}-\frac{12}{h^{2}} e_{4}, \\
\Pi_{6}=e_{1}-\frac{1}{h} e_{3}, \\
\Pi_{7}=e_{1}+\frac{2}{h} e_{3}-\frac{6}{h^{2}} e_{4} \\
\Pi_{8}=e_{1}-\frac{3}{h} e_{3}+\frac{24}{h^{2}} e_{4}-\frac{60}{h^{3}} e_{5}, \\
e_{0}=A e_{1}+A_{d} e_{2}+A_{D} e_{3},
\end{gathered}
$$

and $e_{i} \in \mathbb{R}^{n \times 5 n}$ is defined as $e_{i}=\left[0_{n \times(i-1) n}, I_{n}, 0_{n \times(5-i) n}\right]$ for $i=1,2, \ldots, 5$.

To prove this theorem, Zhao et al., [18], Theorem 1, the following LKF is defined:

$$
V(t)=\sum_{i=1}^{4} V_{i}(t),
$$


where

$$
\begin{aligned}
& V_{1}(t)=\eta^{T}(t) P \eta(t), \\
& V_{2}(t)=\int_{t-h}^{t} x^{T}(s) Q x(s) d s, \\
& V_{3}(t)=h \int_{t-h}^{t} \int_{u}^{t} \dot{x}^{T}(s) S \dot{x}(s) d s d u, \\
& V_{4}(t)=h \int_{t-h}^{t} \int_{u}^{t} \int_{s}^{t} \dot{x}^{T}(s) R \dot{x}(r) d r d s d u, \\
& \eta(t)=\left[x^{T}(t), \int_{t-h}^{t} x^{T}(s) d s, \int_{t-h}^{t} \int_{u}^{t} x^{T}(s) d s d u, \int_{t-h}^{t} \int_{u}^{t} \int_{s}^{t} x^{T}(r) d r d s d u\right]^{T} .
\end{aligned}
$$

Later, they obtained the derivative of this LKF along system of IDDEs (1) as the following:

$$
\dot{V}(t)=\xi^{T}(t) \Psi \xi(t)
$$

where

$$
\xi(t)=\left[x^{T}(t), x^{T}(t-h), \int_{t-h}^{t} x^{T}(s) d s, \int_{t-h}^{t} \int_{u}^{t} x^{T}(s) d s d u, \int_{t-h}^{t} \int_{u}^{t} \int_{s}^{t} x^{T}(r) d r d s d u\right]^{T}
$$

and the matrix $\Psi$ is defined by (4).

\section{US, UAS and Integrability of Solutions}

Consider the un-perturbed system

$$
\dot{x}(t)=A(t) x(t)+A_{d}(t) F(x(t-h))+\int_{t-h}^{t} A_{D}(s) G(x(s)) d s,
$$

which is obtained from system (2).

This section is devoted to improve and generalize the AS result of Zhao et al., [18], Theorem 1. Here, the AS result of Zhao et al., [18], Theorem 1 can be obtained under less conservative conditions and we give additional new results for system (5). The technique used in the proofs is based upon the LKF approach.

A. Assumptions

Throughout this and next sections, the following conditions are needed.

(A1) The functions $F, G \in C\left[\mathbb{R}^{n}, \mathbb{R}^{n}\right]$ satisfy

$$
\|F(x)\| \leq F_{0}\|x\| \text { for all } x \in \mathbb{R}^{n} \text { with } F(0)=0
$$

and

$$
\|G(x)\| \leq G_{0}\|x\| \text { for all } x \in \mathbb{R}^{n} \text { with } G(0)=0,
$$

where $F_{0}$ and $G_{0}$ are positive constants.

(A2) There are constants $F_{0}, G_{0}$ from (A1), $A_{0}>0$ and $h>0$ such that

$$
a_{i i}(t)+\sum_{j=1, j \neq i}^{n}\left|a_{j i}(t)\right| \leq-A_{0} \text { for all } t \in \mathbb{R}^{+}
$$


and

$$
A_{0}-h G_{0}\left\|A_{D}(t)\right\|-F_{0}\left\|A_{d}(t+h)\right\| \geq 0 \text { for all } t \in[-h, \infty) .
$$

(A3) There are constants $A_{0}, G_{0}, F_{0}, h>0$ from (A2) and $K_{0}>0$ such that

$$
a_{i i}(t)+\sum_{j=1, j \neq i}^{n}\left|a_{j i}(t)\right| \leq-A_{0} \text { for all } t \in \mathbb{R}^{+}
$$

and

$$
A_{0}-h G_{0}\left\|A_{D}(t)\right\|-F_{0}\left\|A_{d}(t+h)\right\| \geq K_{0} \text { for all } t \in[-h, \infty) .
$$

The next theorem is the first main result of this paper.

Theorem 2. We assume that conditions (A1) and (A2) are satisfied. Then, the solution $x(t) \equiv 0$ of system (5) is uniformly stable.

Proof. Define a new LKF $\Pi:=\Pi\left(t, x_{t}\right)$ by

$$
\begin{aligned}
\Pi\left(t, x_{t}\right):= & \|x(t)\|+\int_{t-h}^{t}\left\|A_{d}(s+h)\right\|\|F(x(s))\| d s \\
& +\int_{-h}^{0} \int_{t+\eta}^{t}\left\|A_{D}(s)\right\|\|G(x(s))\| d s d \eta,
\end{aligned}
$$

where $-h \leq \eta \leq 0$.

As the next step, it is notable that the KLF $\Pi$ satisfies

$$
\Pi(t, 0)=0, \Pi\left(t, x_{t}\right) \geq\|x\| .
$$

Differentiating the LKF $\Pi$ in (6) along system (5), we find that

$$
\begin{aligned}
\frac{d}{d t} \Pi\left(t, x_{t}\right)= & \sum_{i=1}^{n} x_{i}^{\prime}(t) \operatorname{sgn} x_{i}(t+0) \\
& +\int_{-h}^{0}\left[\left\|A_{D}(t)\right\|\|G(x(t))\|-\left\|A_{D}(t+\eta)\right\|\|G(x(t+\eta))\|\right] d \eta \\
& +\left\|A_{d}(t+h)\right\|\|F(x(t))\|-\left\|A_{d}(t)\right\|\|F(x(t-h))\| \\
= & \sum_{i=1}^{n} x_{i}^{\prime}(t) \operatorname{sgn} x_{i}(t+0) \\
& +h\left\|A_{D}(t)\right\|\|G(x(t))\|-\int_{t-h}^{t}\left\|A_{D}(s)\right\|\|G(x(s))\| d s \\
& +\left\|A_{d}(t+h)\right\|\|F(x(t))\|-\left\|A_{d}(t)\right\|\|F(x(t-h))\| \\
\leq & \sum_{i=1}^{n} x^{\prime}{ }_{i}(t) \operatorname{sgn} x_{i}(t+0)+h G_{0}\left\|A_{D}(t)\right\|\|x(t)\| \\
& -\int_{t-h}^{t}\left\|A_{D}(s)\right\|\|G(x(s))\| d s \\
& +\left\|A_{d}(t+h)\right\|\|F(x(t))\|-\left\|A_{d}(t)\right\|\|F(x(t-h))\| .
\end{aligned}
$$


Using conditions (A1) and (A2), we derive that

$$
\begin{aligned}
\sum_{i=1}^{n} \operatorname{sgn} x_{i}(t+0) x_{i}^{\prime}(t) \leq & \sum_{i=1}^{n} a_{i i}(t)\left|x_{i}(t)\right|+\sum_{i=1}^{n} \sum_{j=1, j \neq i}^{n}\left|a_{j i}(t)\right|\left|x_{i}(t)\right| \\
& +\sum_{i=1}^{n} \sum_{j=1}^{n}\left|a_{d i j}(t)\right|\left|F_{j}(x(t-h))\right| \\
& +\int_{t-h}^{t}\left\|A_{D}(s)\right\|\|G(x(s))\| d s \\
\leq & \sum_{i=1}^{n}\left(a_{i i}(t)+\sum_{j=1, j \neq i}^{n}\left|a_{j i}(t)\right|\right)\left|x_{i}(t)\right| \\
& +\left\|A_{d}(t)\right\|\|F(x(t-h))\|+\int_{t-h}^{t}\left\|A_{D}(s)\right\|\|G(x(s))\| d s \\
\leq & -A_{0}\|x(t)\|+\left\|A_{d}(t)\right\|\|F(x(t-h))\| \\
& +\int_{t-h}^{t}\left\|A_{D}(s)\right\|\|G(x(s))\| d s .
\end{aligned}
$$

A combination of this inequality, inequality (7) and the use of condition of (A1) imply that

$$
\begin{aligned}
\frac{d}{d t} \Pi\left(t, x_{t}\right) \leq & -A_{0}\|x(t)\|+\left\|A_{d}(t)\right\|\|F(x(t-h))\|+\int_{t-h}^{t}\left\|A_{D}(s)\right\|\|G(x(s))\| d s \\
& +h G_{0}\left\|A_{D}(t)\right\|\|x(t)\|-\int_{t-h}^{t}\left\|A_{D}(s)\right\|\|G(x(s))\| d s \\
& +\left\|A_{d}(t+h)\right\|\|F(x(t))\|-\left\|A_{d}(t)\right\|\|F(x(t-h))\| \\
\leq & -A_{0}\|x(t)\|+h G_{0}\left\|A_{D}(t)\right\|\|x(t)\|+F_{0}\left\|A_{d}(t+h)\right\|\|x(t)\| .
\end{aligned}
$$

Hence, we obtain

$$
\frac{d}{d t} \Pi\left(t, x_{t}\right) \leq-\left[A_{0}-h G_{0}\left\|A_{D}(t)\right\|-F_{0}\left\|A_{d}(t+h)\right\|\right]\|x(t)\| .
$$

Using condition (A2), from (8) we obtain

$$
\frac{d}{d t} \Pi\left(t, x_{t}\right) \leq 0
$$

Thus, it is proved that the zero solution of system (5) is uniformly stable.

The next theorem, Theorem 3, is our second main result.

Theorem 3. We assume that conditions (A1) and (A3) are satisfied. Then, the solution $x(t) \equiv 0$ of system (5) is uniformly asymptotically stable. 
Proof. Using condition (A1) and revising the inequality (8) according to condition (A3), we derive that

$$
\begin{aligned}
\Pi\left(t, x_{t}\right):= & \|x(t)\|+\int_{t-h}^{t}\left\|A_{d}(s+h)\right\|\|F(x(s))\| d s \\
& +\int_{-h}^{0} \int_{t+\eta}^{t}\left\|A_{D}(s)\right\|\|G(x(s))\| d s d \eta \\
\leq & \|x(t)\|+F_{0} \int_{t-h}^{t}\left\|A_{d}(s+h)\right\|\|x(s)\| d s \\
& +G_{0} \int_{-h}^{0} \int_{t+\eta}^{t}\left\|A_{D}(s)\right\|\|x(s)\| d s d \eta \\
\leq & \|x(t)\|+h F_{0} \sup _{t-h \leq s \leq t}\left\|A_{d}(s+h)\right\|\|x(s)\| \\
& -h \eta G_{0} \sup _{t+\eta \leq s \leq t}\left\|A_{D}(s)\right\|\|x(s)\|,-h \leq \eta \leq 0, \\
\Pi\left(t, x_{t}\right) \geq & \|x(t)\|
\end{aligned}
$$

and

$$
\begin{aligned}
\frac{d}{d t} \Pi\left(t, x_{t}\right) & \leq-\left[A_{0}-h G_{0} A_{D}(t)-F_{0}\left\|A_{d}(t+h)\right\|\right]\|x(t)\| \\
& \leq-K_{0}\|x(t)\|<0,\|x(t)\| \neq 0 .
\end{aligned}
$$

From the above discussion, it follows that the LKF $\Pi\left(t, x_{t}\right)$ has lower and upper bounds as well as its time derivative along the un-perturbed system (5) is negative definite. From the basic information of qualitative theory of IDDEs, these results are sufficient conditions for all solutions of system (5) uniformly converging to the origin (see, Burton [3], Theorem 4.4.3).

Thus, the solution $x(t) \equiv 0$ of (5) is uniformly asymptotically stable. This is the end of the proof.

The integrability of solutions is given by Theorem 4 at the following.

Theorem 4. We assume that conditions (A1) and (A3) are satiffied. Then, the norms of solutions of system (5) are integrable on $\mathbb{R}^{+}$in sense of Lebesgue.

Proof. Using the LKF $\Pi:=\Pi\left(t, x_{t}\right)$, it follows that (see the proof of Theorem 2)

$$
\frac{d}{d t} \Pi\left(t, x_{t}\right) \leq-K_{0}\|x(t)\| .
$$

From this inequality, an integration gives that

$$
\Pi\left(t, x_{t}\right)-\Pi\left(t_{0}, \phi\left(t_{0}\right)\right) \leq-K_{0} \int_{t_{0}}^{t}\|x(s)\| d s .
$$

Hence, as the next step, we derive that

$$
\int_{t_{0}}^{\infty}\|x(s)\| d s \leq K_{0}^{-1} \Pi\left(t_{0}, \phi\left(t_{0}\right)<+\infty .\right.
$$


The proof is completed.

\section{Numerical Application}

Example 1. We consider the nonlinear delay system

$$
\begin{aligned}
\left(\begin{array}{l}
x_{1}^{\prime}(t) \\
x^{\prime}{ }_{2}(t)
\end{array}\right)= & \left(\begin{array}{cc}
-51+\cos t & 1 \\
1 & -51+\cos t
\end{array}\right)\left(\begin{array}{c}
x_{1}(t) \\
x_{2}(t)
\end{array}\right) \\
& +\left(\begin{array}{cc}
\exp (-t) & 1 \\
1 & \exp (-t)
\end{array}\right)\left(\begin{array}{c}
\frac{x_{1}(t)}{1+x_{1}^{2}\left(t-5^{-1}\right)} \\
\frac{x_{2}(t)}{1+x_{2}^{2}\left(t-5^{-1}\right)}
\end{array}\right) \\
& +\int_{t-\frac{1}{5}}^{t}\left(\begin{array}{cc}
\frac{1}{1+\exp (s)} & 1 \\
2 & \frac{1}{1+\exp (s)}
\end{array}\right)\left(\begin{array}{c}
\frac{x_{1}(t)}{1+x_{1}^{2}(s)} \\
\frac{x_{2}(t)}{1+x_{2}^{2}(s)}
\end{array}\right) d s,
\end{aligned}
$$

where $t \geq 5^{-1}$.

Comparing systems (9) and (5), we obtain the following relations:

$$
\begin{aligned}
& A(t)=\left(\begin{array}{cc}
-51+\cos t & 1 \\
1 & -51+\cos t
\end{array}\right)=\left(\begin{array}{ll}
a_{11}(t) & a_{12}(t) \\
a_{21}(t) & a_{22}(t)
\end{array}\right), \\
& A_{d}(t)=\left(\begin{array}{cc}
\exp (-t) & 1 \\
1 & \exp (-t)
\end{array}\right)=\left(\begin{array}{ll}
a_{d 11}(t) & a_{d 12}(t) \\
a_{d 21}(t) & a_{d 22}(t)
\end{array}\right), \\
& F(x(t-h))=F\left(x_{1}\left(t-5^{-1}\right), x_{2}\left(t-5^{-1}\right)\right)=\left(\begin{array}{c}
\frac{x_{1}(t)}{1+x_{1}^{2}\left(t-5^{-1}\right)} \\
\frac{x_{2}(t)}{1+x_{2}^{2}\left(t-5^{-1}\right)}
\end{array}\right) \\
& G(x)=G\left(x_{1}, x_{2}\right)=\left(\begin{array}{c}
\frac{x_{1}}{1+x_{1}^{2}} \\
\frac{x_{2}}{1+x_{2}^{2}}
\end{array}\right) \text {, where } x=\left(\begin{array}{c}
x_{1} \\
x_{2}
\end{array}\right), \\
& A_{D}(t)=\left(\begin{array}{cc}
\frac{1}{1+\exp (t)} & 1 \\
2 & \frac{1}{1+\exp (t)}
\end{array}\right) .
\end{aligned}
$$

Considering the above relations and proceeding some simple elementary calculations, we have the following estimates:

$$
\begin{gathered}
F(0)=0,\|F(x)\|=\left\|\left(\begin{array}{c}
\frac{x_{1}}{1+x_{1}^{2}\left(t-x^{-1}\right)} \\
\frac{x_{2}}{1+x_{2}^{2}\left(t-5^{-1}\right)}
\end{array}\right)\right\| \\
=\frac{\left|x_{1}\right|}{1+x_{1}^{2}\left(t-5^{-1}\right)}+\frac{\left|x_{2}\right|}{1+x_{2}^{2}\left(t-5^{-1}\right)} \leq F_{0}\|x\|, F_{0}=1, \\
G(0)=0,\|G(x)\|=\left\|\left(\begin{array}{c}
\frac{x_{1}}{1+x_{1}^{2}} \\
\frac{x_{2}}{1+x_{2}^{2}}
\end{array}\right)\right\|=\frac{\left|x_{1}\right|}{1+x_{1}^{2}}+\frac{\left|x_{2}\right|}{1+x_{2}^{2}} \leq G_{0}\|x\|, G_{0}=1, \\
a_{i i}(t)+\sum_{j=1, j \neq i}^{n}\left|a_{j i}(t)\right| \leq-49=-A_{0}
\end{gathered}
$$

since

$$
a_{11}(t)+\left|a_{21}(t)\right|=-51+\cos t+1 \leq-49=-A_{0}
$$

and

$$
a_{22}(t)+\left|a_{12}(t)\right|=-51+\cos t+1 \leq-49=-A_{0},
$$




$$
\begin{gathered}
\left\|A_{d}(t+h)\right\|=\left\|\left(\begin{array}{cc}
\exp (-t-h) & 1 \\
1 & \exp (-t-h)
\end{array}\right)\right\|=\max _{1 \leq j \leq 2} \sum_{i=1}^{2}\left|a_{d i j}(t+h)\right| \\
=\max \{\exp (-t-h)+1,1+\exp (-t-h)\} \\
=1+\exp (-t-h), \text { where } h=\frac{1}{5}, t \geq 5^{-1}, \\
\left\|A_{D}(t)\right\|=\left\|\left(\begin{array}{cc}
\frac{1}{1+\exp (t)} & \frac{1}{2} \\
1+\exp (t)
\end{array}\right)\right\|=\max _{1 \leq j \leq 2} \sum_{i=1}^{2}\left|a_{D i j}(t)\right| \\
=\max \left\{\frac{1}{1+\exp (t)}+2, \frac{1}{1+\exp (t)}+1\right\} \\
=\frac{1}{1+\exp (t)}+2 \leq 3=D_{0} .
\end{gathered}
$$

As for the last step, from these estimates, we arrive that

$$
A_{0}-h G_{0}\left\|A_{D}(t)\right\|-F_{0}\left\|A_{d}(t+h)\right\| \geq 49-5^{-1} \times 3-1-\exp \left(-t-5^{-1}\right)>46=K_{0} .
$$

Thus, we have satisfaction of conditions (A1)-(A3), so the solution $x(t) \equiv 0$ of system (9) is uniformly stable, uniformly asymptotically stable and the norms of the solutions of the system are integrable.

In Figures 1 and 2, the two dimensional system of non-linear DDEs (9) was solved by MATLAB software. The behaviors of stable solutions of system (9) are shown in Figures 1 and 2.

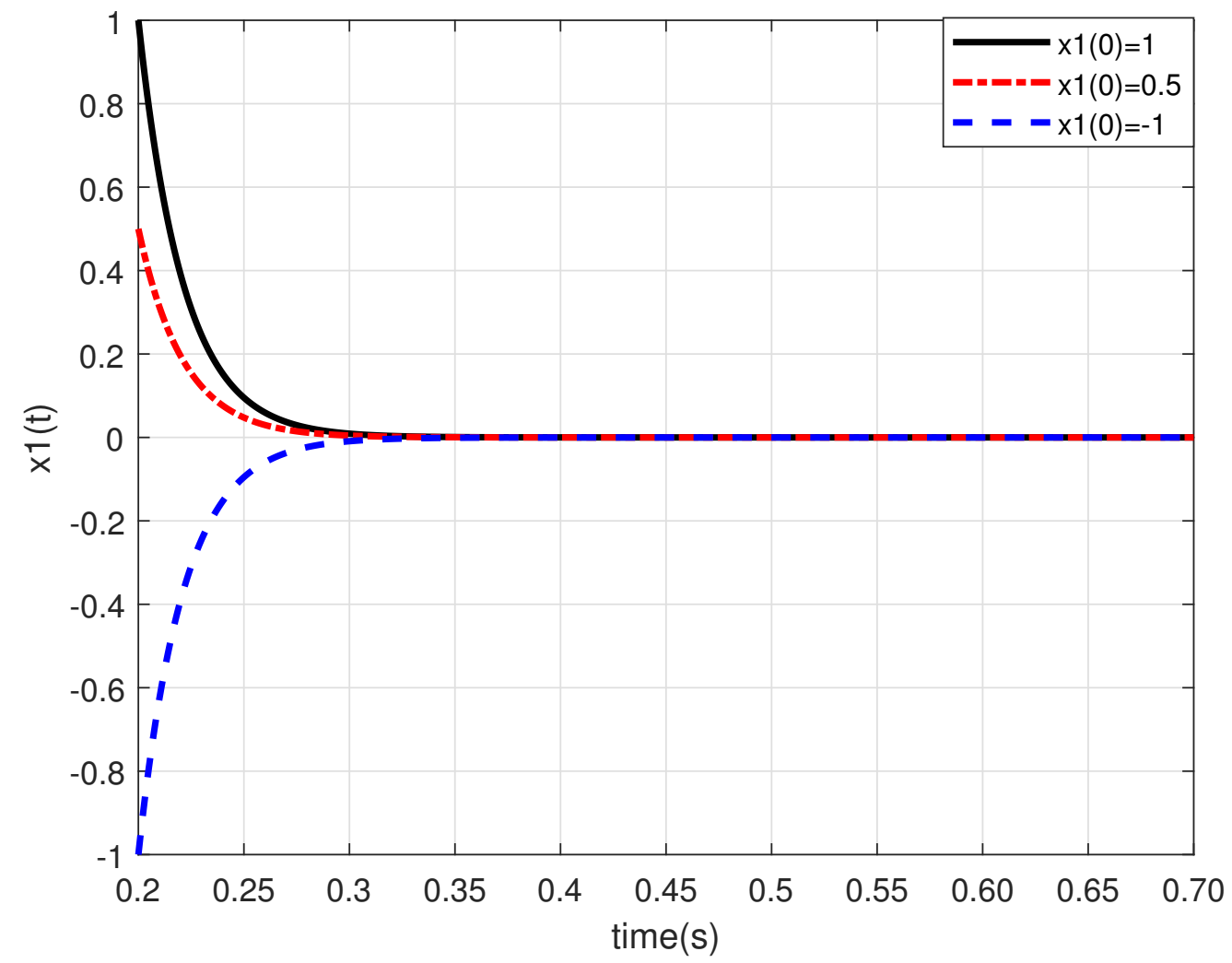

Figure 1. The trajectories of $x_{1}(t)$ of system (9) and their behaviors. 


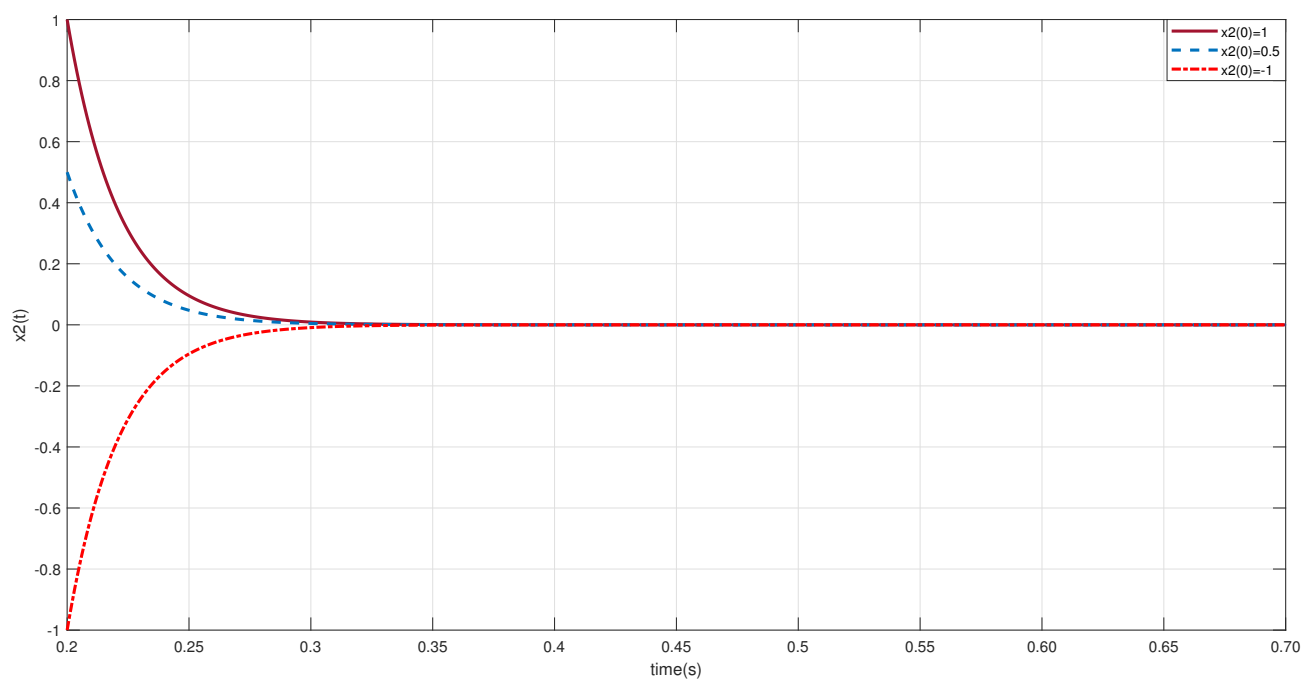

Figure 2. The trajectories of $x_{2}(t)$ of system (9) and their behaviors.

\section{Instability of Solutions}

B. Assumptions

For our instability result, the following conditions are needed.

(A4) Condition (A1) from Theorem 1 is satisfied with

$$
\begin{gathered}
\left\|A_{d}(t+h)\right\| \leq d_{0} \text { for all } t \in \mathbb{R}^{+}, \\
\left\|A_{D}(s)\right\| \leq D_{0} \text { for all } s \in[-h, \infty),
\end{gathered}
$$

where $d_{0}, D_{0}, F_{0}, G_{0}$ and $h$ are positive constants such that

$$
h<\frac{1}{d_{0} F_{0}-\eta D_{0} G_{0}},-h \leq \eta \leq 0 .
$$

(A5) There are constants $F_{0}, G_{0}, h$ from (A2) and $M_{0}>0, \varepsilon>0$ such that

$$
a_{i i}(t)-\sum_{j=1, j \neq i}^{n}\left|a_{j i}(t)\right| \geq M_{0} \text { for all } t \in \mathbb{R}^{+}
$$

and

$$
M_{0}-F_{0}\left\|A_{d}(t+h)\right\|-h G_{0}\left\|A_{D}(t)\right\| \geq \varepsilon .
$$

The next theorem, Theorem 5 , is our instability result.

Theorem 5. We assume that conditions (A4) and (A5) are satisfied. Then, the solution $x(t) \equiv 0$ of system (5) is unstable.

Proof. We define a new LKF $\Pi_{1}:=\Pi_{1}\left(t, x_{t}\right)$ by

$$
\begin{aligned}
\Pi_{1}\left(t, x_{t}\right):= & \|x(t)\|-\int_{t-h}^{t}\left\|A_{d}(s+h)\right\|\|F(x(s))\| d s \\
& -\int_{-h}^{0} \int_{t+\eta}^{t}\left\|A_{D}(s)\right\|\|G(x(s))\| d s d \eta,
\end{aligned}
$$

where $-h \leq \eta \leq 0$. 
Using condition (A4), we obtain from (10) that

$$
\begin{aligned}
\Pi_{1}\left(t, x_{t}\right) & \geq\|x(t)\|-\int_{t-h}^{t} d_{0} F_{0}\|x(s)\| d s-\int_{-h t}^{0} \int_{t+\eta}^{t} D_{0} G_{0}\|x(s)\| d s d \eta \\
& \geq\|x(t)\|-h d_{0} F_{0} \sup _{t-h \leq s \leq t}\|x(s)\|+h \eta D_{0} G_{0} \sup _{t+\eta \leq s \leq t}\|x(s)\| \\
& \geq\|x(t)\|-h\left(d_{0} F_{0}-\eta D_{0} G_{0}\right) \sup _{t-h \leq s \leq t}\|x(s)\| \\
& =\left[1-h\left(d_{0} F_{0}-\eta D_{0} G_{0}\right)\right] \sup _{t-h \leq s \leq t}\|x(s)\|>0
\end{aligned}
$$

provided $\|x(t)\|=\sup _{t-h \leq s \leq t}\|x(s)\|, h<\frac{1}{d_{0} F_{0}-\eta D_{0} G_{0}},-h \leq \eta \leq 0$ and $\|x(t)\| \neq 0$.

Next, the derivative of LKF $\Pi_{1}$ in (10) along solutions of system (5) gives that

$$
\begin{aligned}
\frac{d}{d t} \Pi_{1}\left(t, x_{t}\right)= & \sum_{i=1}^{n} x_{i}^{\prime}(t) \operatorname{sgn} x_{i}(t+0) \\
& -\left\|A_{d}(t+h)\right\|\|F(x(t))\|+\left\|A_{d}(t)\right\|\|F(x(t-h))\| \\
& -h\left\|A_{D}(t)\right\|\|G(x(t))\|+\int_{t-h}^{t}\left\|A_{D}(s)\right\|\|G(x(s))\| d s .
\end{aligned}
$$

Using condition (A4), for the first term of (11), we obtain

$$
\begin{aligned}
\sum_{i=1}^{n} \operatorname{sgn} x_{i}(t+0) x_{i}^{\prime}(t) \geq & \sum_{i=1}^{n} a_{i i}(t)\left|x_{i}(t)\right|-\sum_{i=1}^{n} \sum_{j=1, j \neq i}^{n}\left|a_{j i}(t)\right|\left|x_{i}(t)\right| \\
& -\sum_{i=1}^{n} \sum_{j=1}^{n}\left|a_{d i j}(t)\right|\left|F_{j}(x(t-h))\right| \\
& -\int_{t-h}^{t}\left\|A_{D}(s)\right\|\|G(x(s))\| d s \\
\geq & \sum_{i=1}^{n}\left(a_{i i}(t)-\sum_{j=1, j \neq i}^{n}\left|a_{j i}(t)\right|\right)\left|x_{i}(t)\right| \\
& -\left\|A_{d}(t)\right\|\|F(x(t-h))\|-\int_{t-h}^{t}\left\|A_{D}(s)\right\|\|G(x(s))\| d s \\
\geq & M_{0}\|x(t)\|-\left\|A_{d}(t)\right\|\|F(x(t-h))\| \\
& -\int_{t-h}^{t}\left\|A_{D}(s)\right\|\|G(x(s))\| d s .
\end{aligned}
$$


On gathering the relations (11), (12) and using conditions (A4) and (A5), we have

$$
\begin{aligned}
\frac{d}{d t} \Pi_{1}\left(t, x_{t}\right) \geq & M_{0}\|x(t)\|-\left\|A_{d}(t)\right\|\|F(x(t-h))\|-\int_{t-h}^{t}\left\|A_{D}(s)\right\|\|G(x(s))\| d s \\
& -\left\|A_{d}(t+h)\right\|\|F(x(t))\|+\left\|A_{d}(t)\right\|\|F(x(t-h))\| \\
& -h\left\|A_{D}(t)\right\|\|G(x(t))\|+\int_{t-h}^{t}\left\|A_{D}(s)\right\|\|G(x(s))\| d s \\
= & M_{0}\|x(t)\|-\left\|A_{d}(t+h)\right\|\|F(x(t))\|-h\left\|A_{D}(t)\right\|\|G(x(t))\| \\
\geq & M_{0}\|x(t)\|-F_{0}\left\|A_{d}(t+h)\right\|\|x(t)\|-h G_{0}\left\|A_{D}(t)\right\|\|x(t)\| \\
= & {\left[M_{0}-F_{0}\left\|A_{d}(t+h)\right\|-h G_{0}\left\|A_{D}(t)\right\|\right]\|x(t)\| } \\
\geq & \varepsilon\|x(t)\|>0,\|x(t)\| \neq 0 .
\end{aligned}
$$

As a result of the above study, we conclude that the solution $\left(\begin{array}{l}x_{1}(t) \\ x_{2}(t)\end{array}\right)=\left(\begin{array}{l}0 \\ 0\end{array}\right)$ of system (5) is unstable provided conditions (A4) and (A5) are satisfied.

\section{Numerical Application}

Example 2. Let us consider the following nonlinear delay system:

$$
\begin{aligned}
\left(\begin{array}{l}
x_{1}^{\prime}(t) \\
x_{2}^{\prime}(t)
\end{array}\right)= & \left(\begin{array}{cc}
51-\cos t & 1 \\
1 & 51-\cos t
\end{array}\right)\left(\begin{array}{c}
x_{1}(t) \\
x_{2}(t)
\end{array}\right) \\
& +\left(\begin{array}{cc}
\exp (-t) & 1 \\
1 & \exp (-t)
\end{array}\right)\left(\begin{array}{c}
\frac{x_{1}(t)}{1+x_{1}^{2}\left(t-5^{-1}\right)} \\
\frac{x_{2}(t)}{1+x_{2}^{2}\left(t-5^{-1}\right)}
\end{array}\right) \\
& +\int_{t-\frac{1}{5}}^{t}\left(\begin{array}{cc}
\frac{1}{1+s^{2}+\exp (t)} & 1 \\
2 & \frac{1}{1+s^{2}+\exp (t)}
\end{array}\right)\left(\begin{array}{c}
\frac{x_{1}(s)}{1+x_{1}^{2}(s)} \\
\frac{x_{2}(s)}{1+x_{2}^{2}(s)}
\end{array}\right) d s,
\end{aligned}
$$

where $t \geq 5^{-1}$.

Comparing systems (13) and (5), it follows that

$$
A(t)=\left(\begin{array}{cc}
51-\cos t & 1 \\
1 & 51-\cos t
\end{array}\right)=\left(\begin{array}{ll}
a_{11}(t) & a_{12}(t) \\
a_{21}(t) & a_{22}(t)
\end{array}\right) .
$$

Next, the matrices $A_{d}(t), A_{D}(t)$ and the functions $G\left(x_{1}, x_{2}\right), F\left(x_{1}\left(t-5^{-1}\right), x_{2}\left(t-5^{-1}\right)\right)$ are the same as those given by Example 1. As for the following step, it is verified that

$$
a_{i i}(t)-\sum_{j=1, j \neq i}^{n}\left|a_{j i}(t)\right|=51-\cos t-1>48=M_{0}
$$

since

$$
a_{11}(t)-\left|a_{21}(t)\right|=51-\cos t-1>48=M_{0}
$$

and

$$
a_{22}(t)-\left|a_{12}(t)\right|=51-\cos t-1>48=M_{0} .
$$

As for the next step, from the above estimates and those in Example 1, we obtain that

$$
\begin{gathered}
h=\frac{1}{5}<\frac{1}{2+\frac{3}{5}} \leq \frac{1}{d_{0} F_{0}-\eta D_{0} G_{0}} \text {, with } \eta=-\frac{1}{5}, \\
M_{0}-F_{0}\left\|A_{d}(t+h)\right\|-h G_{0}\left\|A_{D}(t)\right\|
\end{gathered}
$$




$$
\geq 48-2-5^{-1} \times 3>45=\varepsilon .
$$

Therefore, the solution $\left(\begin{array}{l}x_{1}(t) \\ x_{2}(t)\end{array}\right)=\left(\begin{array}{l}0 \\ 0\end{array}\right)$ of system (13) is unstable.

In Figures 3 and 4, the two dimensional system of non-linear DDEs (13) was solved by MATLAB software. The behaviors of unstable solutions of system (13) are shown in Figures 3 and 4.

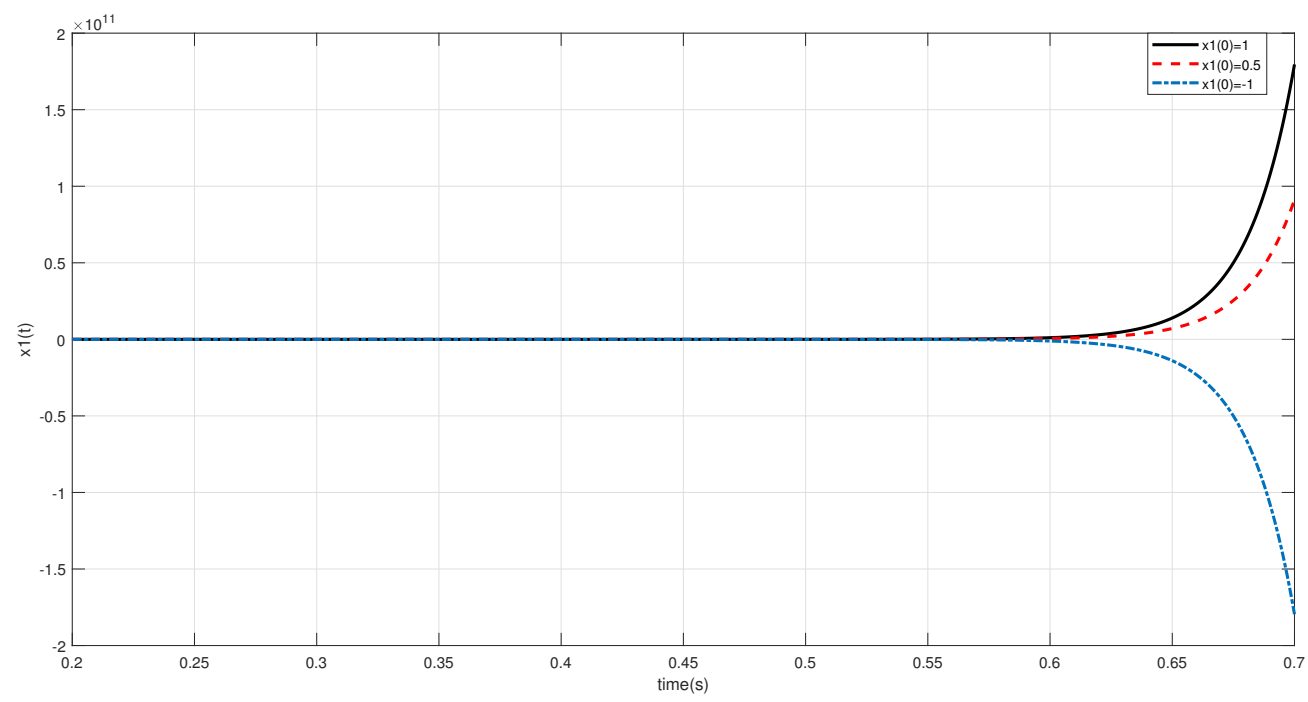

Figure 3. The trajectories of $x_{1}(t)$ of system (13) and their behaviors.

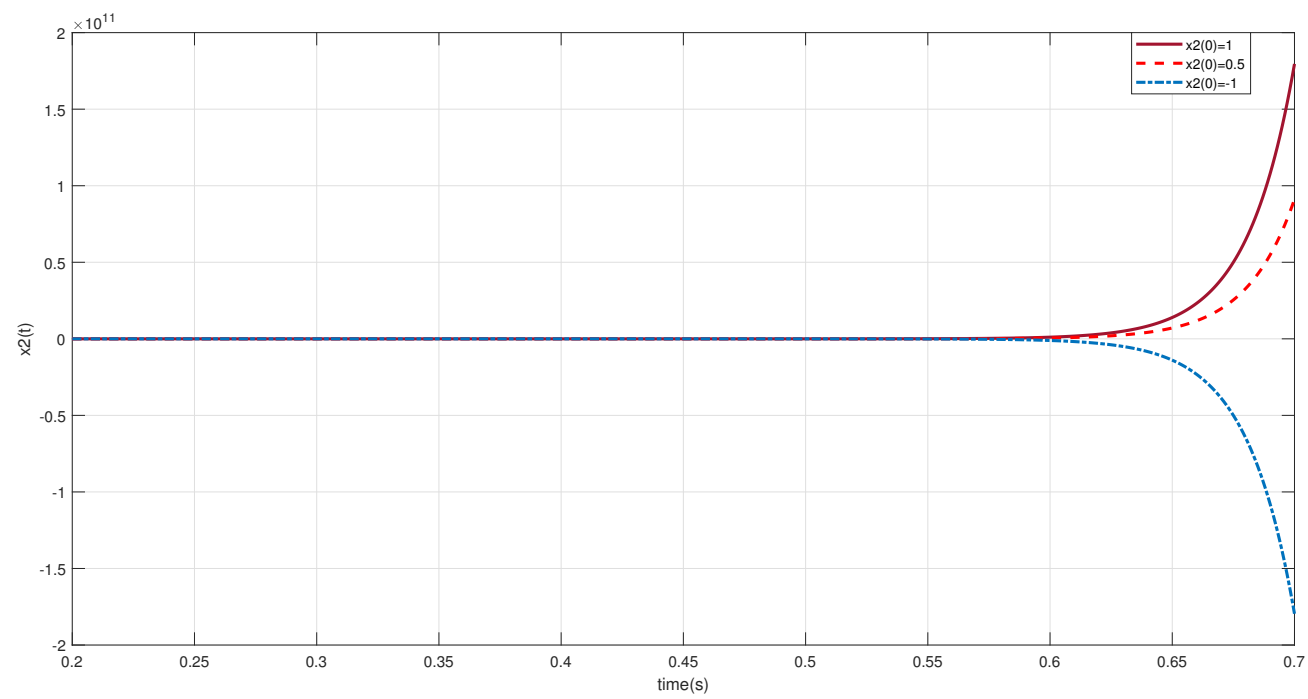

Figure 4. The trajectories of $x_{2}(t)$ of system (13) and their behaviors.

\section{Boundedness}

We consider the perturbed system (2) and give a new theorem on the boundedness of solutions.

For the boundedness result of this paper, we need the following additional assumption. C. Assumption

(A6) The function $H \in C\left(\mathbb{R}^{+} \times \mathbb{R}^{n} \times \mathbb{R}^{n}\right.$, $\left.\mathbb{R}^{n}\right)$ satisfies

$$
\|H(t, x(t), x(t-h))\| \leq\left|H_{0}(t)\right|\|x(t)\|
$$


for all $t \in \mathbb{R}^{+}$and for all $x, x(t-h) \in \mathbb{R}^{n}$, where $H_{0} \in C\left(\mathbb{R}^{+}, \mathbb{R}\right)$, the function $H_{0}$ is bounded and there are constants $F_{0}, G_{0}, A_{0}>0$ and $h>0$ from (A2) such that

$$
a_{i i}(t)+\sum_{j=1, j \neq i}^{n}\left|a_{j i}(t)\right| \leq-A_{0} \text { for all } t \in \mathbb{R}^{+}
$$

and

$$
A_{0}-h G_{0}\left\|A_{D}(t)\right\|-F_{0}\left\|A_{d}(t+h)\right\|-\left|H_{0}(t)\right| \geq 0 \text { for all } t \in[-h, \infty) .
$$

The following theorem, Theorem 6 , is the boundedness result of this paper.

Theorem 6. The solutions of perturbed system (2) are bounded at infinity if conditions (A1) and (A6) are satisfied

Proof. In this proof, we use again the LKF given by (6). Clearly, using conditions (A1) and (A6), one can find that

$$
\begin{aligned}
\frac{d}{d t} \Pi\left(t, x_{t}\right) & \leq-\left[A_{0}-F_{0}\left\|A_{d}(t+h)\right\|-h G_{0}\left\|A_{D}\right\|\right]\|x(t)\|+\|H(t, x(t), x(t-h))\| \\
& \leq-\left[A_{0}-F_{0}\left\|A_{d}(t+h)\right\|-h G_{0}\left\|A_{D}\right\|-\left|H_{0}(t)\right|\right]\|x(t)\| \leq 0 .
\end{aligned}
$$

As a result of this inequality, we find

$$
\Pi\left(t, x_{t}\right) \leq \Pi\left(t_{0}, \phi\left(t_{0}\right)\right) .
$$

Hence, it is notable from the definition of LKF (6) that

$$
\|x(t)\| \leq \Pi\left(t_{0}, \phi\left(t_{0}\right)\right), t \geq t_{0} .
$$

If $t \rightarrow+\infty$, then

$$
\lim _{t \rightarrow+\infty}\||x(t)|\| \leq \lim _{t \rightarrow+\infty} \Pi\left(t_{0}, \phi\left(t_{0}\right)\right)=\Pi\left(t_{0}, \phi\left(t_{0}\right)\right) \equiv \text { a positive constant. }
$$

Thus, the solutions of system (2) are bounded as $t \rightarrow+\infty$. The proof of Theorem 6 is completed.

\section{Numerical Application}

Example 3. We now take into consideration the following nonlinear delay system:

$$
\begin{aligned}
\left(\begin{array}{l}
x^{\prime}{ }_{1}(t) \\
x^{\prime}{ }_{2}(t)
\end{array}\right)= & \left(\begin{array}{cc}
-51+\cos t & 1 \\
1 & -51+\cos t
\end{array}\right)\left(\begin{array}{c}
x_{1}(t) \\
x_{2}(t)
\end{array}\right) \\
& +\left(\begin{array}{cc}
\exp (-t) & 1 \\
1 & \exp (-t)
\end{array}\right)\left(\begin{array}{c}
\frac{x_{1}(t)}{1+x_{1}^{2}\left(t-5^{-1}\right)} \\
\frac{x_{2}(t)}{1+x_{2}^{2}\left(t-5^{-1}\right)}
\end{array}\right) \\
& +\int_{t-\frac{1}{5}}^{t}\left(\begin{array}{cc}
\frac{1}{1+\exp (s)} & 1 \\
2 & \frac{1}{1+\exp (s)}
\end{array}\right)\left(\begin{array}{c}
\frac{x_{1}(s)}{1+x_{1}^{2}(s)} \\
\frac{x_{2}(s)}{1+x_{2}^{2}(s)}
\end{array}\right) d s \\
& +\left(\begin{array}{c}
\frac{\sin x_{1}(t)}{1+\exp (t) \exp \left(\left|x_{1}\left(t-5^{-1}\right)\right|\right)} \\
\frac{\sin x_{2}(t)}{1+\exp (t)+\exp \left(\left|x_{2}\left(t-5^{-1}\right)\right|\right)}
\end{array}\right) .
\end{aligned}
$$


Comparing systems (14) and (2), it follows that

$$
\begin{aligned}
H\left(t, x_{1}, x_{2}, x_{1}\left(t-5^{-1}\right), x_{2}\left(t-5^{-1}\right)\right) & =\left(\begin{array}{c}
\frac{\sin x_{1}}{1+\exp (t)+\exp \left(\left|x_{1}\left(t-5^{-1}\right)\right|\right)} \\
\frac{\sin x_{2}}{1+\exp (t)+\exp \left(\left|x_{2}\left(t-5^{-1}\right)\right|\right)}
\end{array}\right) \\
\left\|H\left(t, x_{1}, x_{2}, x_{1}\left(t-5^{-1}\right), x_{2}\left(t-5^{-1}\right)\right)\right\| & \leq \frac{\left|\sin x_{1}\right|}{1+\exp (t)+\exp \left(\left|x_{1}\left(t-5^{-1}\right)\right|\right)} \\
& +\frac{\left|\sin x_{2}\right|}{1+\exp (t)+\exp \left(\left|x_{2}\left(t-5^{-1}\right)\right|\right)} \\
& \leq \frac{\left|\sin x_{1}\right|+\left|\sin x_{2}\right|}{1+\exp (t)} \\
& \leq \frac{\left|x_{1}\right|+\left|x_{2}\right|}{1+\exp (t)} \\
& =\frac{1}{1+\exp (t)}\|x\|, \text { where }\left|H_{0}(t)\right|=\frac{1}{1+\exp (t)} .
\end{aligned}
$$

Next, the matrices $A(t), A_{d}(t), A_{D}(t)$ and the functions $G\left(x_{1}, x_{2}\right), F\left(x_{1}\left(t-5^{-1}\right), x_{2}(t-\right.$ $\left.\left.5^{-1}\right)\right)$ are the same as those in Example 1. Hence, it is seen that

$$
A_{0}-h G_{0}\left\|A_{D}(t)\right\|-F_{0}\left\|A_{d}(t+h)\right\|-\left|H_{0}(t)\right| \geq 49-5^{-1} \times 3-2-\frac{1}{1+\exp (t)}>0 .
$$

Then, it is obvious that all solutions $\left(\begin{array}{l}x_{1}(t) \\ x_{2}(t)\end{array}\right)$ of system (14) are bounded.

In Figures 5 and 6, the two dimensional system of non-linear DDEs (14) was solved by MAT$L A B$ software. The behaviors of boundedness solutions of system (14) are shown in Figures 5 and 6.

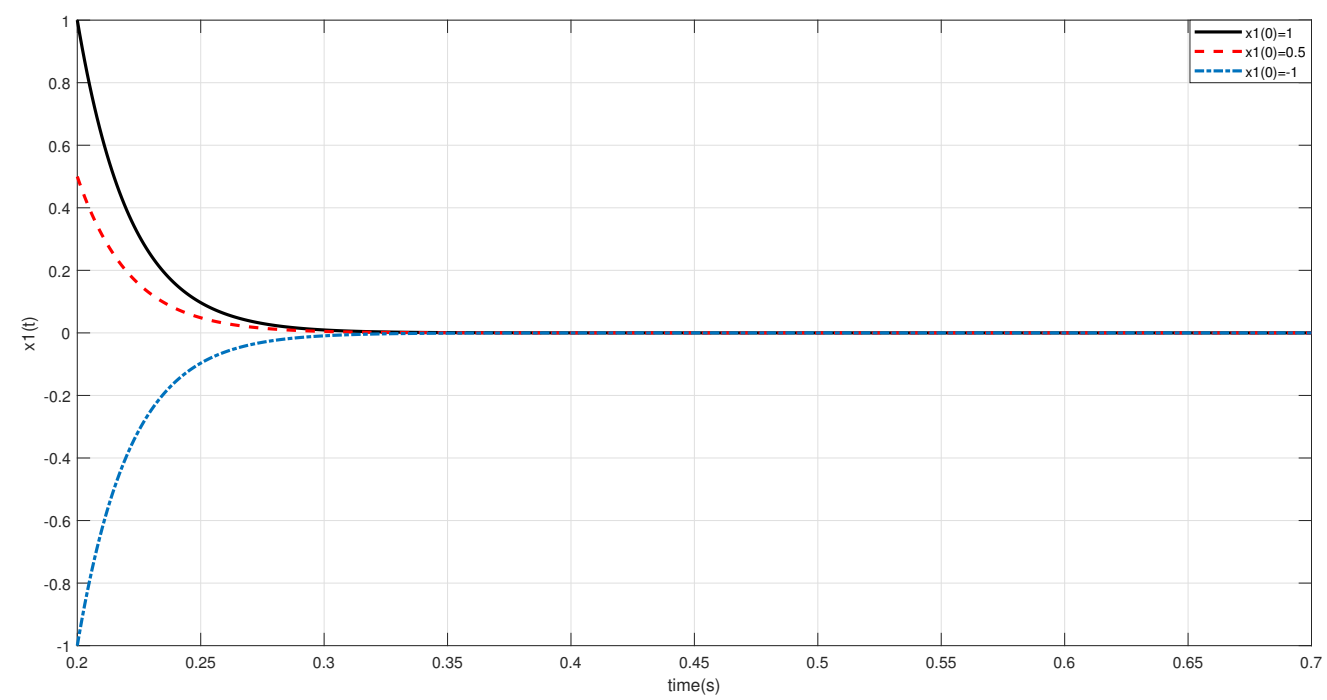

Figure 5. The trajectories of $x_{1}(t)$ of system (14) and their behaviors. 


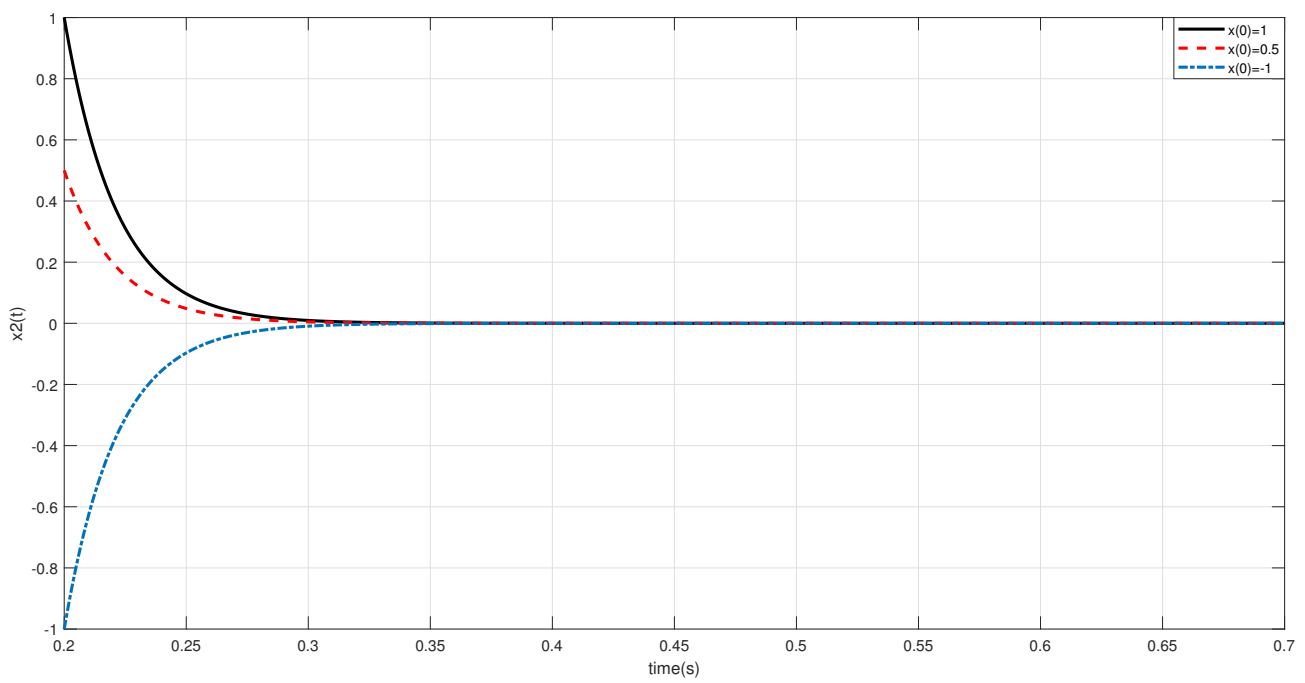

Figure 6. The trajectories of $x_{2}(t)$ of system (14) and their behaviors.

Remark 1. In the three previous sections, Sections 4, 6 and 8, called "Numerical application", to illustrate Theorems 2-5 and to show their applications in particular cases, Examples 1-3 were solved using the 4th order Runge-Kutta method via MATLAB Simulink software by taking the step size as 0.001 .

\section{Contributions}

Here, we would like to summarize the contributions and novelty of this paper and to explain comparisons with some former literature.

(1) The system of IDDEs considered in Zhao et al., [18], system (1), is linear. System (1) also has a simple form. However, perturbed and unperturbed systems of IDDEs (2) and (5), respectively, are non-linear and they have more general forms than system (1). Indeed, instead of the constant matrices $A, A_{d}, A_{D}$ and linear term $x(t-h)$ of (1), we took the variable matrices $A(t), A_{d}(t), A_{D}(s)$ and the non-linear functions $F(x(t-h))$, $G(x)$ in both of systems (2) and (5). We also added the nonlinear perturbed term $H(t, x(t), x(t-h))$ to system (2). These mean that system (2) and (5) extend and improve system (1) from linear case to the nonlinear case.

(2) Zhao et al., [18], Theorem 1 proved a nice result on the asymptotic stability of system (1) (see the above Theorem 1) by using a very interesting approach. Indeed, the authors used the new double integral inequality in terms of LMIs and the LKF (see Section 2) to prove [18], Theorem 1. However, the use of double integral inequality, LMIs and the LKF can cause stronger conditions for the asymptotic stability result of [18], Theorem 1. This case can be seen when we look at the conditions of [18], Theorem 1. In this paper, we did not use the double integral inequality, LMIs and the LKF of [18], Theorem 1. From this point of view, we defined two new, different, interesting and effective LKFs (see the LKFs given by (6) and (12)) and used these two LKFs. Here, the LKFs (6), (12) can lead very less conservative, more suitable and practicable conditions. This case can be checked clearly when we compare the LKF used by Zhao et al., [18], Theorem 1 (see, Section 2) and the LKF (6) used to prove the uniform asymptotic stability result of this paper, Theorem 3. 
Indeed, to the best of information, up to now, the LKFs

$$
\begin{aligned}
\Pi\left(t, x_{t}\right):= & \|x(t)\|+\int_{t-h}^{t}\left\|A_{d}(s+h)\right\|\|F(x(s))\| d s \\
& +\int_{-h}^{0} \int_{t+\eta}^{t}\left\|A_{D}(s)\right\|\|G(x(s))\| d s d \eta .
\end{aligned}
$$

and

$$
\begin{aligned}
\Pi_{1}\left(t, x_{t}\right):= & \|x(t)\|-\int_{t-h}^{t}\left\|A_{d}(s+h)\right\|\|F(x(s))\| d s \\
& -\int_{-h}^{0} \int_{t+\eta}^{t}\left\|A_{D}(s)\right\|\|G(x(s))\| d s d \eta .
\end{aligned}
$$

were not used in the literature to discuss the fundamental behaviors of solutions related to IDDEs and DDEs. Next, indeed, as it is well-known, the investigation of fundamental behaviors of trajectories of non-linear systems of IDDEs and DDEs is a difficult task. However, the effective roles of the LKFs (6) and (12) come from the integral terms

$$
\int_{t-h}^{t}\left\|A_{d}(s+h)\right\|\|F(x(s))\| d s, \int_{-h t+\eta}^{0} \int_{t}^{t}\left\|A_{D}(s)\right\|\|G(x(s))\| d s d \eta
$$

and

$$
-\int_{t-h}^{t}\left\|A_{d}(s+h)\right\|\|F(x(s))\| d s \text { and } \int_{-h}^{0} \int_{t+\eta}^{t}\left\|A_{D}(s)\right\|\|G(x(s))\| d s d \eta .
$$

Without loss of generality, if suitable LKFs could be found, then very less conservative qualitative criteria can be obtained.

(3) The UAS result of this paper, Theorem 3, extends and improves the result of Zhao et al., [18], Theorem 1 under weaker and more suitable conditions. Next, indeed, UAS implies US, but its converse is not true. Additionally, to the best of information, there is no result on the instability of system of IDDEs (5). Theorem 5 is the first instability result in the literature. The uniform stability and integrability results of unperturbed system (5) and boundedness result of perturbed system (2), i.e., Theorems 2, 4 and 6, respectively, are new and original. These theorems have also very suitable and less conservative conditions for proper and related applications in various fields of sciences and engineering.

Next, for the cases of US and UAS, since

$$
A_{0}-F_{0}\left\|A_{d}(t+h)\right\|>0 \text { (see (A2)), }
$$

the upper bound for the constant delay term $h$ is determined as the following, respectively:

$$
h \leq \frac{A_{0}-F_{0}\left\|A_{d}(t+h)\right\|}{G_{0}\left\|A_{D}(t)\right\|} \text { and } h<\frac{A_{0}-F_{0}\left\|A_{d}(t+h)\right\|}{G_{0}\left\|A_{D}(t)\right\|},\left\|A_{D}(t)\right\| \neq 0 .
$$

As for the case of the instability, since

$$
\begin{gathered}
M_{0}-F_{0}\left\|A_{d}(t+h)\right\|-h G_{0}\left\|A_{D}(t)\right\|>0(\text { see (A5)), } \\
h<\frac{1}{d_{0} F_{0}-\eta D_{0} G_{0}},-h \leq \eta \leq 0,(\text { see (A4)) }
\end{gathered}
$$


the upper bound for the constant delay term $h$ is determined as the following:

$$
h=\min \left\{\frac{M_{0}-F_{0}\left\|A_{d}(t+h)\right\|}{G_{0}\left\|A_{D}(t)\right\|}, \frac{1}{d_{0} F_{0}-\eta D_{0} G_{0}}\right\},-h \leq \eta \leq 0 .
$$

Finally, for the case of boundedness, since

$$
A_{0}-F_{0}\left\|A_{d}(t+h)\right\|-\left|H_{0}(t)\right|>0,
$$

it follows that

$$
h \leq \frac{A_{0}-F_{0}\left\|A_{d}(t+h)\right\|-\left|H_{0}(t)\right|}{G_{0}\left\|A_{D}(t)\right\|},\left\|A_{D}(t)\right\| \neq 0,
$$

where the function $H_{0}(t)$ is bounded such that

$$
\left|H_{0}(t)\right| \leq B_{0} \text { with } A_{0}-F_{0}\left\|A_{d}(t+h)\right\|-B_{0}>0 .
$$

(4) In this paper, three examples, Examples 1-3, are provided, which satisfy the conditions of the related theorems. These examples are solved by the 4th order Runge-Kutta method in MATLAB software.

\section{Conclusions}

In this article, we have discussed the US, UAS, integrability, instability and boundedness problems of un-perturbed and perturbed systems of non-linear IDDEs, respectively. Five new theorems have been obtained and the obtained theorems are new and original with scientific novelties. The proofs of these theorems are performed by defining two new LKFs. Three examples are given as numerical applications of our theorems. We believe that the results of this paper provide new and effective contributions to the literature and may be useful to researchers working on these concepts.

Author Contributions: Conceptualization, O.T., C.T. and J.-C.Y.; Data curation, O.T., C.T.; Formal analysis, O.T., C.T., Y.W. and J.-C.Y.; Funding acquisition, Y.W.; Methodology, C.T., O.T. and Y.W.; Project administration, J.-C.Y.; Supervision, Y.W. and J.-C.Y.; Validation, O.T., C.T.; Visualization, O.T.; Writing-original draft, O.T. All authors have read and agreed to the published version of the manuscript.

Funding: This research is supported by financial grants from the National Natural Science Foundation of China (No.: 12171435).

Institutional Review Board Statement: Not applicable.

Informed Consent Statement: Not applicable.

Data Availability Statement: Not applicable.

Conflicts of Interest: The authors declare no conflicts of interest.

\section{References}

1. Bohner, M.; Tunç, O.; Tunç, C. Qualitative analysis of Caputo fractional integro-differential equations with constant delays. Comp. Appl. Math. 2021, 40, 214. [CrossRef]

2. Berezansky, L.; Diblík, J.; Svoboda, Z.; Šmarda, Z. Uniform exponential stability of linear delayed integro-differential vector equations. J. Differ. Equations 2021, 270, 573-595. [CrossRef]

3. Burton, T.A. Volterra Integral and Differential Equations; 2nd ed.; Mathematics in Science and Engineering, 202; Elsevier: Amsterdam, The Netherlands, 2005.

4. Graef, J. R.; Tunç, C. Continuability and boundedness of multi-delay functional integro-differential equations of the second order. RACSAM 2015, 109, 169-173. [CrossRef]

5. Hale, J. K. ; Verduyn Lunel, S.M. Introduction to Functional-Differential Equations; Applied Mathematical Sciences, 99; Springer: New York, NY, USA, 1993. [CrossRef]

6. Huyen, D.T.K.; Yao, J.-C. Solution stability of a linearly perturbed constraint system and applications. Set-Valued Var. Anal. 2019, 27, 169-189. [CrossRef] 
7. Liu, X.; Zhao, D. New stability criterion for time-delay systems via an augmented Lyapunov-Krasovskii functional. Appl. Math. Lett. 2021, 116, 7. [CrossRef]

8. Nieto, J.J.; Tunç, O. An application of Lyapunov-Razumikhin method to behaviors of Volterra integro-differential equations. RACSAM 2021, 115, 197. [CrossRef]

9. Raffoul, Y. Exponential stability and instability in finite delay nonlinear Volterra integro-differential equations. Dyn. Contin. Discrete Impuls. Syst. Ser. A Math. Anal. 2013, 1,95-106.

10. Tian, J.; Ren, Z.; Zhong, S. A new integral inequality and application to stability of time-delay systems. Appl. Math. Lett. 2020, 101, 7. [CrossRef]

11. Tian, Y.; Wang, Z. A new multiple integral inequality and its application to stability analysis of time-delay systems. Appl. Math. Lett. 2020, 105, 8. [CrossRef]

12. Tunç, C.; Tunç, O. New qualitative criteria for solutions of Volterra integro-differential equations. Arab. J. Basic Appl. Sci. 2018, 3, 158-165. [CrossRef]

13. Tunç, C.; Tunç, O. On the stability, integrability and boundedness analyses of systems of integro-differential equations with time-delay retardation. RACSAM 2021, 115, 1-17. [CrossRef]

14. Tunç, O. On the behaviors of solutions of systems of non-linear differential equations with multiple constant delays. $R A C S A M$ 2021, 115, 164,

15. Tunç, O.; Atan, Ö.; Tunç, C.; Yao, J.-C. Qualitative analyses of integro-fractional differential equations with Caputo derivatives and retardations via the Lyapunov-Razumikhin Method. Axioms 2021, 10, 58. [CrossRef]

16. Xu, X.; Liu, L.; Feng, G. Stability and stabilization of infinite delay systems: A Lyapunov-based approach. IEEE Trans. Automat. Control 2020, 11, 4509-4524. [CrossRef]

17. Wang, Q. The stability of a class of functional differential equations with infinite delays. Ann. Differ. Equ. 2000, 1, 89-97.

18. Zhao, N.; Lin, C.; Chen, B.; Wang, Q.G. A new double integral inequality and application to stability test for time-delay systems. Appl. Math. Lett. 2017, 65, 26-31. [CrossRef]

19. Zhao, J.; Meng, F. Stability analysis of solutions for a kind of integro-differential equations with a delay. Math. Probl. Eng. 2018, 2018,9519020 ,

20. Zeng, H.B.; He, Y.; Wu, M.; Jinhua, S. New results on stability analysis for systems with discrete distributed delay. Autom. J. IFAC 2015, 60, 189-192. [CrossRef]

21. Zhu, X.L.; Yang, G.H. New stability criteria for systems with time-varying delay. In Proceedings of the 2008 Chinese Control and Decision Conference, Yantai, China, 2-4 July 2008. 